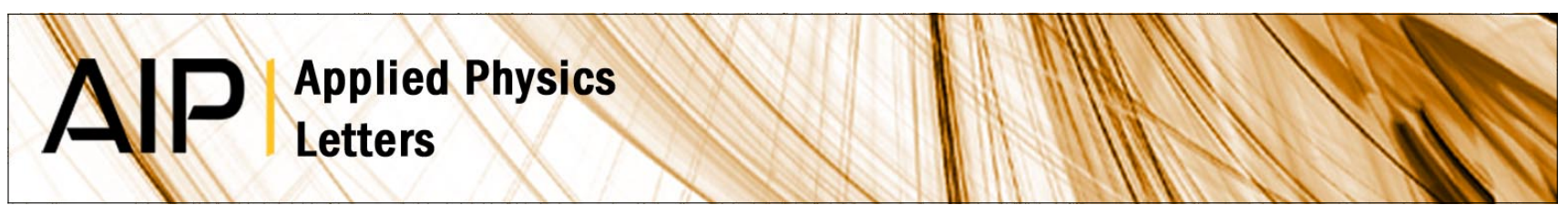

\title{
Lutetium-doped EuO films grown by molecular-beam epitaxy
}

A. Melville, T. Mairoser, A. Schmehl, D. E. Shai, E. J. Monkman et al.

Citation: Appl. Phys. Lett. 100, 222101 (2012); doi: 10.1063/1.4723570

View online: http://dx.doi.org/10.1063/1.4723570

View Table of Contents: http://apl.aip.org/resource/1/APPLAB/v100/i22

Published by the American Institute of Physics.

Additional information on Appl. Phys. Lett.

Journal Homepage: http://apl.aip.org/

Journal Information: http://apl.aip.org/about/about_the_journal

Top downloads: http://apl.aip.org/features/most_downloaded

Information for Authors: http://apl.aip.org/authors

\section{ADVERTISEMENT}

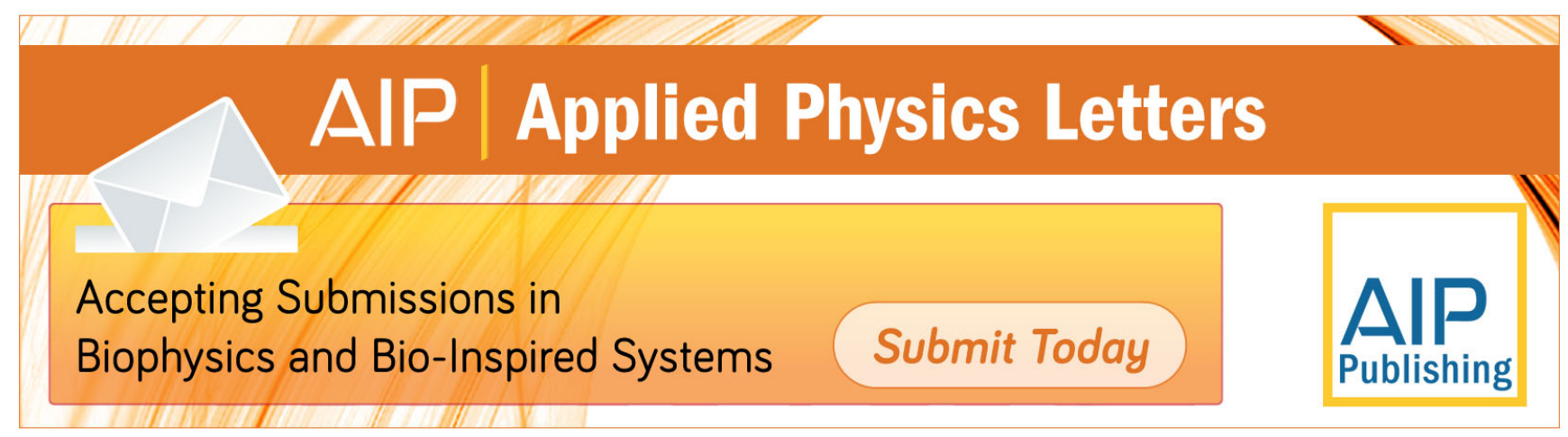




\title{
Lutetium-doped EuO films grown by molecular-beam epitaxy
}

\author{
A. Melville, ${ }^{1}$ T. Mairoser, ${ }^{2}$ A. Schmehl, ${ }^{2}$ D. E. Shai, ${ }^{3}$ E. J. Monkman, ${ }^{3}$ J. W. Harter, ${ }^{3}$ \\ T. Heeg, ${ }^{1}$ B. Holländer, ${ }^{4}$ J. Schubert, ${ }^{4}$ K. M. Shen, ${ }^{3,5}{ }^{1}$ J. Mannhart, ${ }^{6}$ and D. G. Schlom ${ }^{1,5}$ \\ ${ }^{1}$ Department of Materials Science and Engineering, Cornell University, Ithaca, New York 14853, USA \\ ${ }^{2}$ Zentrum für elektronische Korrelationen und Magnetismus, Universität Augsburg, Universitätsstraße 1, \\ D-86159 Augsburg, Germany \\ ${ }^{3}$ Laboratory of Atomic and Solid State Physics, Department of Physics, Cornell University, Ithaca, New York \\ 14853, USA \\ ${ }^{4}$ Peter Grünberg Institute, PGI 9-IT, JARA-FIT, Research Centre Jülich, D-52425 Jülich, Germany \\ ${ }^{5}$ Kavli Institute at Cornell for Nanoscale Science, Ithaca, New York 14853, USA \\ ${ }^{6}$ Max Planck Institute for Solid State Research, D-70569 Stuttgart, Germany
}

(Received 24 January 2012; accepted 10 May 2012; published online 29 May 2012)

The effect of lutetium doping on the structural, electronic, and magnetic properties of epitaxial $\mathrm{EuO}$ thin films grown by reactive molecular-beam epitaxy is experimentally investigated. The behavior of Lu-doped $\mathrm{EuO}$ is contrasted with doping by lanthanum and gadolinium. All three dopants are found to behave similarly despite differences in electronic configuration and ionic size. Andreev reflection measurements on Lu-doped EuO reveal a spin-polarization of $96 \%$ in the conduction band, despite non-magnetic carriers introduced by 5\% lutetium doping. (C) 2012 American Institute of Physics. [http://dx.doi.org/10.1063/1.4723570]

The ferromagnetic half-metal europium oxide $(\mathrm{EuO})$ has potential for spin-based devices like spin-injectors based on its $>90 \%$ spin-polarization $^{1}$ or optical devices based on its giant magneto-optic Kerr effect ${ }^{2}$ and a Faraday rotation of $8.5 \times 10^{5} \mathrm{deg} / \mathrm{cm}$ in a field of $2 \mathrm{~T} .^{3}$ Its low Curie temperature $\left(T_{\mathrm{C}}=69 \mathrm{~K}\right),{ }^{4}$ however, impedes the incorporation of $\mathrm{EuO}$ into devices.

The $T_{\mathrm{C}}$ of EuO can be increased by doping it with electrons. The interaction between the Eu $f$-electrons and the dopant electrons enhances the ferromagnetic exchange energy ${ }^{4,5}$ and results in an increased $T_{\mathrm{C}}$. To date, this has been accomplished through the use of trivalent cations including iron, ${ }^{6-8}$ lanthanum, ${ }^{1,9,10}$ gadolinium,,${ }^{1-17}$ and holmium. ${ }^{9}$ Alternatively, the $T_{\mathrm{C}}$ can be increased by deliberately making oxygen-deficient EuO such that the resulting oxygen vacancies donate an electron. ${ }^{6,18-22}$ In the cationdoped $\mathrm{EuO}$ films, an unknown and uncontrolled concentration of oxygen vacancies is often included, which may be responsible for the disparate results for the dependence of $T_{\mathrm{C}}$ on cation doping. For example, in films doped with gadolinium, the maximum reported $T_{\mathrm{C}}$ varies between $120 \mathrm{~K}$ (Ref. 15) and $170 \mathrm{~K} .{ }^{14}$ Films doped with lanthanum have a maximum reported $T_{\mathrm{C}}$ between $118 \mathrm{~K}$ (Ref. 1) and $200 \mathrm{~K} .{ }^{10}$ Likewise, for films doped with iron, the reported $T_{\mathrm{C}}$ varies between $88 \mathrm{~K}$ and $200 \mathrm{~K} .{ }^{6,7}$ For Gd-doped films grown in an adsorption-controlled regime, however, the Curie temperatures are consistent and similar, ${ }^{15,16}$ conceivably due to a minimized amount of oxygen vacancies realized in this particular growth regime. ${ }^{23}$ In this letter, we report the behavior of an unexplored dopant for EuO, lutetium, which enhances the $T_{\mathrm{C}}$ in epitaxial films grown in an adsorption-controlled regime. We contrast the magnetic and electronic properties of $\mathrm{EuO}$ doped with lanthanum, gadolinium, and lutetium when grown under identical adsorption-controlled growth conditions.

Doped EuO thin films were grown directly on (110) $\mathrm{YAlO}_{3}$ substrates via reactive oxide molecular-beam epitaxy
(Veeco 930 and GEN10) in an adsorption-controlled growth regime at a substrate temperature of $350{ }^{\circ} \mathrm{C}$ to ensure high crystalline quality and stoichiometric films. ${ }^{17,23}$ Pure oxygen gas was introduced during growth to a background oxygen partial pressure of $1 \times 10^{-9}$ Torr above the base pressure of the vacuum system. In the adsorption-controlled regime, the oxygen flux limits the EuO growth rate if the incident flux of europium metal is greater than the effective oxygen flux. The adsorption-controlled growth regime and the growth rate of $\mathrm{EuO}$ were determined by measuring the areal density of Eu atoms in calibration EuO samples using Rutherford backscattering spectrometry (RBS) ${ }^{23}$ Each film was nominally $35 \mathrm{~nm}$ thick, but the uncertainty in the exact oxygen flux during each growth corresponds to roughly $10 \%$ variability in the EuO growth rate and therefore the thickness. Europium and the dopant were codeposited from separate effusion cells. Prior to growth, the europium flux was calibrated using a quartz crystal microbalance (QCM) to a flux of $1.1 \times 10^{14}$ atoms $/\left(\mathrm{cm}^{2} \times \mathrm{s}\right)$. This flux was $100 \%$ higher than the rate at which europium was incorporated into the $\mathrm{EuO}$ film. The lanthanum, gadolinium, and lutetium fluxes were calibrated by a QCM to correspond to $4 \%$ doping of the $\mathrm{EuO}$ for the films characterized by in situ x-ray photoelectron spectroscopy (XPS) measurements and 5\% for the films characterized ex situ.

Several films were transferred under ultra-high vacuum immediately after growth into an analysis chamber for XPS characterization using $\mathrm{Mg} K_{\alpha}$ radiation $(1253.6 \mathrm{eV})$. The remaining films were capped with $20 \mathrm{~nm}$ of amorphous silicon to protect the films from further oxidation during their $e x$ situ characterization. Structural characterization was performed using four-circle X-ray diffraction (XRD) utilizing $\mathrm{Cu} K_{\alpha}$ radiation. The $T_{\mathrm{C}}$ was determined using superconducting quantum-interference-device (SQUID) magnetometry. The films were measured in zero field after applying a demagnetization routine at $300 \mathrm{~K}$ to minimize the spurious magnetic fields originating from parts of the SQUID. ${ }^{16}$ At 
this temperature the $\mathrm{EuO}$ is in the paramagnetic state without oriented ferromagnetic domains. Bridges were patterned into the doped EuO films using photolithography in combination with in situ ion etching and sputter deposition. ${ }^{16}$ Bridges $1 \mathrm{~mm} \times 0.1 \mathrm{~mm}$ in size were used for four-point resistivity measurements from $4 \mathrm{~K}$ to $300 \mathrm{~K}$ and Hall measurements at $4 \mathrm{~K}$, and bridges $50 \mu \mathrm{m} \times 250 \mu \mathrm{m}$ in size were used across a ramp-type junction between superconducting niobium and metallic Lu-doped $\mathrm{EuO}$ for differential four-point conductivity measurements for Andreev reflection from $1.8 \mathrm{~K}$ to $12 \mathrm{~K}$.

The effect of the three dopants on the crystallinity of the epitaxial doped EuO films was assessed by XRD. Figure 1 shows the $\theta-2 \theta$ XRD patterns of typical EuO films doped with $5 \%$ lanthanum, gadolinium, or lutetium. All samples exhibit only $00 \ell \mathrm{EuO}$ peaks in addition to the substrate peaks. XRD rocking curves show the full width at half maximum (FWHM) of the EuO 002 peaks to be $0.16 \pm 0.01^{\circ}$ for all samples. These data indicate that the doped EuO samples are structurally indistinguishable by XRD.

SQUID magnetometry reveals the $T_{\mathrm{C}}$ of the $5 \%$ doped films to be similar. La-doped $\mathrm{EuO}$ has $T_{\mathrm{C}}=116 \mathrm{~K}$; Gddoped $\mathrm{EuO}$ has $T_{\mathrm{C}}=122 \mathrm{~K}$; and Lu-doped $\mathrm{EuO}$ has $T_{\mathrm{C}}=119 \mathrm{~K}$ as seen in Fig. 2(a). The observed kink in the Ludoped $\mathrm{EuO}$ (and to a lesser extent, the La-doped $\mathrm{EuO}$ ) could be consistent with clustering of dopants in the film. ${ }^{10}$ The temperature dependence of the resistivity for the same samples is shown in Fig. 2(b). Hall measurements for these samples reveal that $\mathrm{Gd}$-doped $\mathrm{EuO}$ has the highest carrier concentration with $n=5 \times 10^{20} \mathrm{~cm}^{-3}$ followed by both the La- and the Lu-doped $\mathrm{EuO}$ with carrier concentrations of $n=2.1 \times 10^{20} \mathrm{~cm}^{-3}$ and $n=1.8 \times 10^{20} \mathrm{~cm}^{-3}$, respectively. The difference in $T_{\mathrm{C}}$ and carrier concentration is within the accuracy of our doping level control, the accuracy of the film thickness, and the strong dependence of dopant activation on substrate temperature. ${ }^{1}$

XAS was used to verify the $3^{+}$oxidation state of the gadolinium in the Gd-doped $\mathrm{EuO}$ films ${ }^{17}$ and XPS was used to verify the $3^{+}$oxidation state of the lanthanum and lutetium in the La- and Lu-doped EuO films, respectively. XAS and XPS were also used to confirm that the oxidation state of $\mathrm{Eu}$ is nearly completely $\mathrm{Eu}^{2+}$, with a small amount

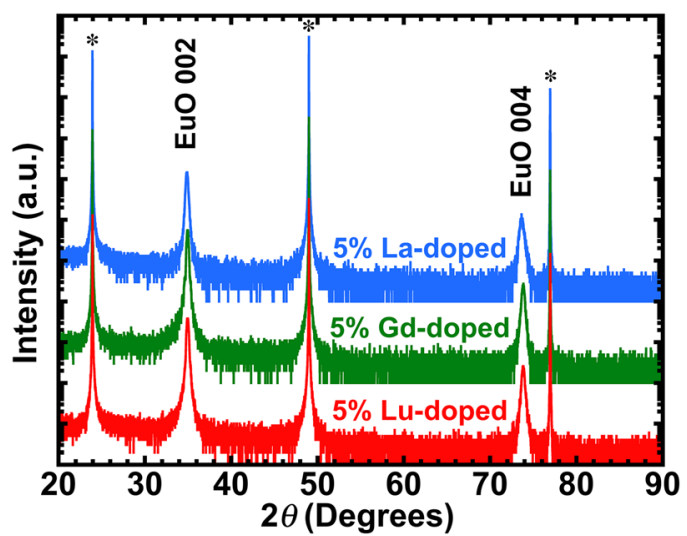

FIG. 1. $\theta-2 \theta$ scans comparing epitaxial EuO films with $5 \%$ lanthanumdoping (blue), 5\% gadolinium-doping (green), and 5\% lutetium-doping (red). All three films are phase-pure with no indication of dopant insolubility. The curves are offset for clarity and the substrate peaks are marked with asterisks.
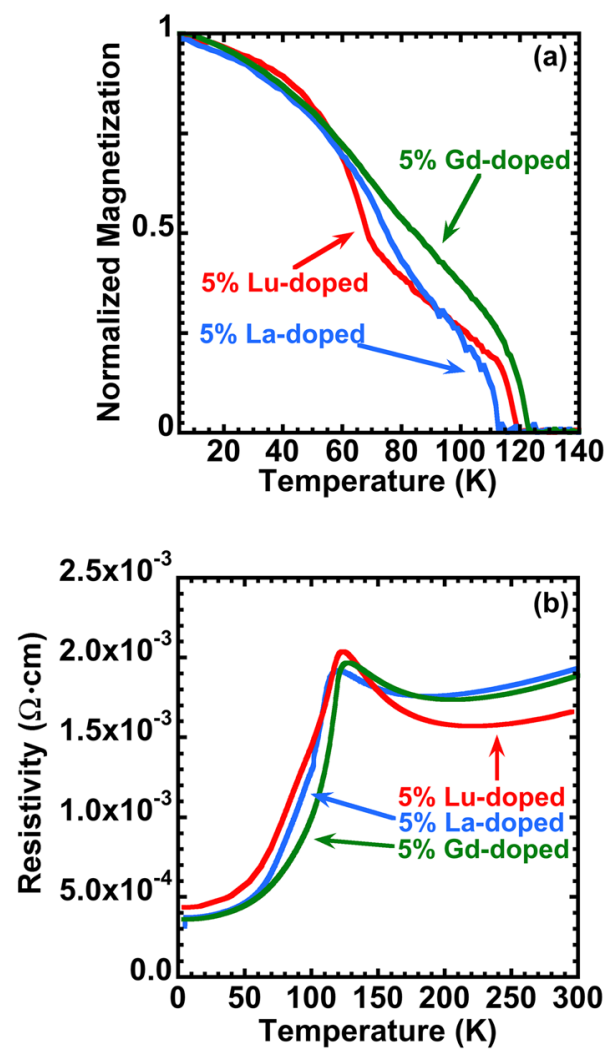

FIG. 2. (a) Normalized magnetization as a function of temperature of the same three samples studied in Fig. 1. All three dopants increase the $T_{\mathrm{C}}$ to approximately the same value at 5\% doping concentration. (b) Resistivity as a function of temperature for the same three samples. Doped EuO exhibits reduced resistivity and a reduced metal-to-insulator transition compared to undoped EuO. The change in resistivity is comparable regardless of dopant choice.

of $\mathrm{Eu}^{3+}$ attributed to surface oxidation in uncapped samples as documented in the supplementary information of Ref. 16. Figure 3 shows the XPS intensity of the lutetium $4 \mathrm{~d}$ core-level multiplets for the lutetium in Lu-doped EuO, lutetium metal, and $\mathrm{Lu}_{2} \mathrm{O}_{3}$. Lutetium metal was deposited at room temperature in vacuum by $\mathrm{MBE}$, and $\mathrm{Lu}_{2} \mathrm{O}_{3}$ was formed by keeping the lutetium metal in vacuum with a background pressure of $2 \times 10^{-9}$ Torr for at least $1 \mathrm{~h}$. Comparing the peak positions to the literature ${ }^{16}$ confirmed

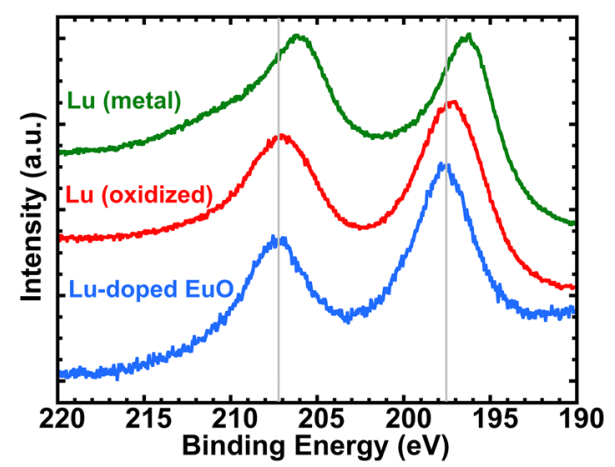

FIG. 3. Comparison of X-ray photoemission intensity of the lutetium $4 \mathrm{~d}$ core-level multiplets between 4\% Lu-doped EuO (blue), lutetium metal (green), and oxidized lutetium (red). The dashed lines are guides for the eye to highlight the similarity in peak position between the $4 \% \mathrm{Lu}$-doped EuO and the oxidized lutetium. 

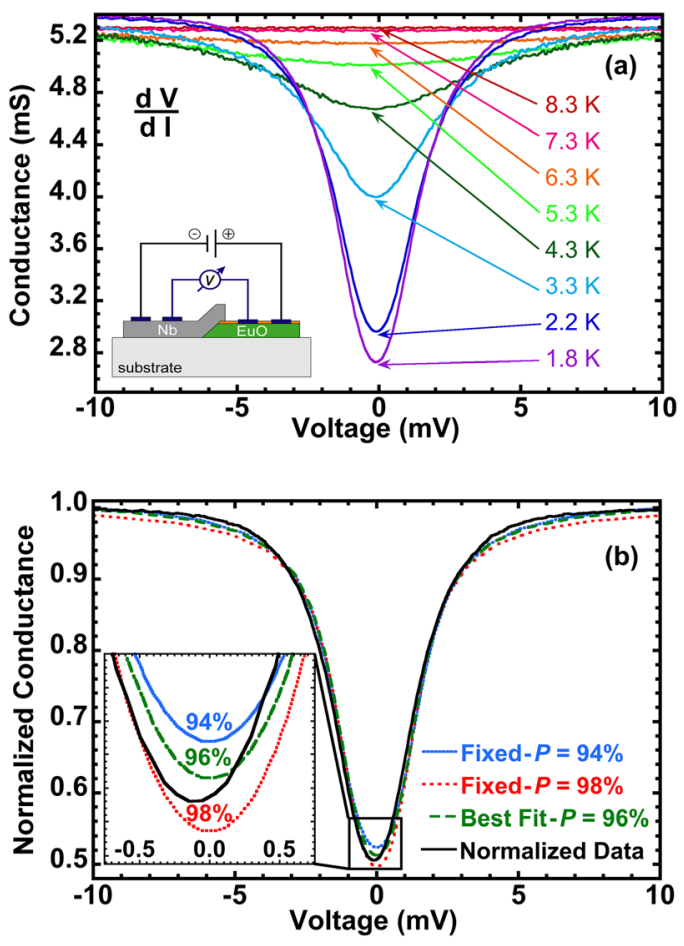

FIG. 4. Andreev reflection measurements of the contact between a niobium electrode and the same 5\% Lu-doped sample studied in Figs. 1 and 2. (a) Conductance vs. voltage characteristics were measured at different temperatures. Below the superconducting gap of the niobium electrode, the conductance diminishes because Andreev reflection is forbidden for an interface between a 100\% spin-polarized material and a superconductor. The inset shows a schematic of the ramp-type junction used for differential four-point conductivity measurements. (b) Fits to the $1.8 \mathrm{~K}$ measurement using the Blonder-Tinkham-Klapwijk model are in accordance with a spinpolarization of $96 \%$ (green). For comparison, fits are shown that fix $P$ to $94 \%$ (blue) and to $98 \%$ (red). The inset shows the overlap of the curves near zero voltage bias to better illustrate the match of the best fit. The best fit parameters were $Z=0.9, \Delta=1.28 \mathrm{meV}, R=77$, and $P=96 \%$.

that the lutetium in the Lu-doped $\mathrm{EuO}$ film was indeed $3^{+}$ as expected. A similar process was followed for identifying the oxidation state of lanthanum in the La-doped EuO. From the free carrier concentration, oxidation state, and the doping concentration, it is evident rare-earth dopant ions inject far less than one mobile electron into the $\mathrm{EuO}$ conduction band. The dopant activation is less than $40 \%$, in agreement with results for $\mathrm{Gd}$-doped $\mathrm{EuO},{ }^{24}$ pointing to the possible ubiquity of the challenge of achieving high dopant activation in EuO. All samples have comparable resistivity curves, reduced dopant activation, and fairly similar carrier concentrations.

Differential conductance measurements were performed on 5\% Lu-doped $\mathrm{EuO}$ and are shown in Fig. 4. Since Andreev reflection is suppressed at the interface between a material with high spin-polarization and a superconducting material, a drop in conductivity across the Lu-doped $\mathrm{EuO} /$ $\mathrm{Nb}$ interface for energies less than the superconducting gap of the niobium film at temperatures below the superconducting critical temperature is expected. By fitting the drop in conductance to the Blonder-Tinkham-Klapwijk model ${ }^{25}$ that has been modified specifically for non-negligible series resistance in spin-polarized ferromagnetic semiconductor devices, ${ }^{26-28}$ one can extract the spin-polarization value. The best fit is in accordance with a spin-polarization of $96 \%$, which is in agreement with previous reports of the near-complete spin-polarization of EuO. ${ }^{1}$ The high spinpolarization despite 5\% doping of nonmagnetic atoms is critically important, as it shows that EuO retains its high spin-polarization in the presence of a dopant which significantly boosts its $T_{\mathrm{C}}$. This is not so surprising given that doped $\mathrm{EuO}$ is a half-metal due to the spin-splitting of the conduction band, ${ }^{29-31}$ so the carriers are spin-polarized anyway despite the nature of the dopant.

In summary, we have explored the properties of $\mathrm{Lu}^{-}$ doped EuO. Lutetium donates electrons to $\mathrm{EuO}$ in the same fashion as lanthanum and gadolinium dopants. Furthermore, EuO retains near-complete spin-polarization $(P=96 \%)$ despite being heavily doped with the non-magnetic ion $\mathrm{Lu}^{3+}$.

The work at Cornell was supported by the AFOSR (Grant No. FA9550-10-1-0123), the NSF MRSEC program by cooperative agreement 1120296, and NSF DMR0847385, and a Cottrell Scholars Award (20025). The work in Augsburg was supported by the DFG (Grant No. TRR 80) and the EC (oxIDes). AM gratefully acknowledges support from the NSF IGERT program (NSF Award DGE-0654193) and by the IMI Program of the National Science Foundation under Award No. DMR 0843934. EJM acknowledges NSERC for PGS support.

${ }^{1}$ A. Schmehl, V. Vaithyanathan, A. Herrnberger, S. Thiel, C. Richter, M. Liberati, T. Heeg, M. Röckerath, L. F. Kourkoutis, S. Mühlbauer, P. Böni, D. A. Muller, Y. Barash, J. Schubert, Y. Idzerda, J. Mannhart, and D. G. Schlom, Nat. Mater. 6, 882-887 (2007).

${ }^{2}$ J. C. Suits and K. Lee, J. Appl. Phys. 42, 3258-3260 (1971).

${ }^{3}$ K. Y. Ahn and M. W. Shafer, J. Appl. Phys. 41, 1260-1262 (1970).

${ }^{4}$ T. R. McGuire and M. W. Shafer, J. Appl. Phys. 35, 984-988 (1964).

${ }^{5}$ F. Holtzberg, T. R. McGuire, and S. Methfessel, Phys. Rev. Lett. 13, 18-21 (1964).

${ }^{6}$ K. Lee and J. C. Suits, Phys. Lett. 34A, 141-142 (1971).

${ }^{7}$ T. R. McGuire, G. F. Petrich, B. L. Olson, V. L. Moruzzi, and K. Y. Ahn, J. Appl. Phys. 42, 1775-1777 (1971).

${ }^{8}$ K. Y. Ahn, K. N. Tu, and W. Reuter, J. Appl. Phys. 42, 1769-1770 (1971).

${ }^{9}$ M. W. Shafer and T. R. McGuire, J. Appl. Phys. 39, 588-590 (1968).

${ }^{10}$ H. Miyazaki, H. J. Im, K. Terashima, S. Yagi, M. Kato, K. Soda, T. Ito, and S. Kimura, Appl. Phys. Lett. 96, 232503 (2010).

${ }^{11}$ S. von Molnár and M. W. Shafer, J. Appl. Phys. 41, 1093-1094 (1970).

${ }^{12}$ A. A. Samokhvalov, B. A. Gizhevskii, and M. I. Simonova, Sov. Phys. Solid State 14, 230-231 (1972).

${ }^{13}$ A. Mauger, C. Godart, M. Escorne, J. C. Achard, and J. P. Desfours, J. Phys. (Paris) 39, 1125-1133 (1978).

${ }^{14}$ H. Ott, S. Heise, R. Sutarto, Z. Hu, C. Chang, H. Hsieh, H.-J. Lin, C. Chen, and L. Tjeng, Phys. Rev. B. 73, 094407 (2006).

${ }^{15}$ R. Sutarto, S. Altendorf, B. Coloru, M. Moretti Sala, T. Haupricht, C. Chang, Z. Hu, C. Schüßler-Langeheine, N. Hollmann, H. Kierspel, J. Mydosh, H. Hsieh, H.-J. Lin, C. Chen, and L. Tjeng, Phys. Rev. B. 80, 085308 (2009).

${ }^{16}$ T. Mairoser, A. Schmehl, A. Melville, T. Heeg, L. Canella, P. Böni, W. Zander, J. Schubert, D. Shai, E. Monkman, K. Shen, D. Schlom, and J. Mannhart, Phys. Rev. Lett. 105, 257206 (2010).

${ }^{17}$ T. Mairoser, A. Schmehl, A. Melville, T. Heeg, W. Zander, J. Schubert, D. E. Shai, E. J. Monkman, K. M. Shen, T. Z. Regier, D. G. Schlom, and J. Mannhart, Appl. Phys. Lett. 98, 102110 (2011).

${ }^{18}$ K. Lee and J. C. Suits, IEEE Trans. Magn. 7, 391 (1971).

${ }^{19}$ Y. Capiomont, Nguyen-Van-Dang, O. Massenet, and B. K.Chakraverty, Solid State Commun. 10, 679-683 (1972).

${ }^{20}$ O. Massenet, Y. Capiomont, and N. Van Dang, J. Appl. Phys. 45, 3593-3599 (1974).

${ }^{21}$ H. Miyazaki, T. Ito, H. Im, K. Terashima, S. Yagi, M. Kato, K. Soda, and S.-I. Kimura, Jpn. J. Appl. Phys. 48, 055504 (2009). 
${ }^{22}$ M. Barbagallo, N. Hine, J. Cooper, N.-J. Steinke, A. Ionescu, C. Barnes, C. Kinane, R. Dalgliesh, T. Charlton, and S. Langridge, Phys. Rev. B. 81, 235216 (2010).

${ }^{23}$ R. W. Ulbricht, A. Schmehl, T. Heeg, J. Schubert, and D. G. Schlom, Appl. Phys. Lett. 93, 102105 (2008).

${ }^{24}$ W. C. Lang, B. D. Padalia, L. M. Watson, D. J. Fabian, and P. R. Norris, Faraday Discuss. Chem. Soc. 60, 37-43 (1975).

${ }^{25}$ G. E. Blonder, M. Tinkham, and T. M. Klapwijk, Phys. Rev. B. 21, 4515-4532 (1982).
${ }^{26}$ S. Upadhyay, A. Palanisami, R. N. Louie, and R. A. Buhrman, Phys. Rev. Lett. 81, 3247-3250 (1998).

${ }^{27}$ R. J. Soulen, Jr., Science 282, 85-88 (1998).

${ }^{28}$ I. I. Mazin, Phys. Rev. Lett. 83, 1427-1430 (1999).

${ }^{29}$ S. Von Molnár, IBM IBM J. Res. Dev. 14, 269-275 (1970).

${ }^{30}$ M. R. Oliver, J. O. Dimmock, and T. B. Reed, IBM J. Res. Develop. 14, 276-278 (1970).

${ }^{31}$ P. Steeneken, L. Tjeng, I. Elfimov, G. Sawatzky, G. Ghiringhelli, N. Brookes, and D.-J. Huang, Phys. Rev. Lett. 88, 047201 (2002). 